\title{
Generalized Dunkl-Williams inequality in 2-inner product spaces
}

Abbas Najati ${ }^{1}$, M Mohammadi Saem ${ }^{1}$ and Jae-Hyeong Bae 2* $^{*}$

"Correspondence: jhbae@khu.ac.kr ${ }^{2}$ Graduate School of Education, Kyung Hee University, Yongin, 446-701, Republic of Korea

Full list of author information is available at the end of the article

\begin{abstract}
We consider the generalized Dunkl-Williams inequality in 2-normed spaces. Also, we give necessary and sufficient conditions for having the equality case in the strictly convex 2-normed space $X$.
\end{abstract}

Keywords: 2-normed spaces; 2-inner product spaces; strictly convex; Dunkl-William inequality; Maligranda inequality

\section{Introductions and preliminaries}

In 1964, Dunkl and Williams [1] proved that if $x, y$ are non-zero vectors in a normed linear space $X$, then

$$
\left\|\frac{x}{\|x\|}-\frac{y}{\|y\|}\right\| \leq \frac{4\|x-y\|}{\|x\|+\|y\|} .
$$

Also, after that it was proved that the equality holds if and only if $x=y$.

Maligranda [2] obtained a refinement of the Dunkl-Williams inequality. He proved that if $x, y$ are non-zero vectors in a normed linear space $X$, then

$$
\begin{aligned}
& \left\|\frac{x}{\|x\|}-\frac{y}{\|y\|}\right\| \leq \frac{|\|x\|-\|y\||+\|x-y\|}{\max (\|x\|,\|y\|)}, \\
& \left\|\frac{x}{\|x\|}-\frac{y}{\|y\|}\right\| \geq \frac{\|x-y\|-\|\| x\|-\| y \| \mid}{\min (\|x\|,\|y\|)} .
\end{aligned}
$$

Also, the Maligranda inequality and its reverse in normed linear spaces were proved by Mercer in [3]. In [4] Kato et al. improved the triangle inequality and provided the reverse by showing that

$$
\begin{aligned}
& \left\|\sum_{j=1}^{n} x_{j}\right\|+\left(n-\left\|\sum_{j=1}^{n} \frac{x_{j}}{\left\|x_{j}\right\|}\right\|\right) \min _{1 \leq i \leq n}\left\|x_{i}\right\| \leq \sum_{j=1}^{n}\left\|x_{j}\right\|, \\
& \left\|\sum_{j=1}^{n} x_{j}\right\|+\left(n-\left\|\sum_{j=1}^{n} \frac{x_{j}}{\left\|x_{j}\right\|}\right\|\right) \max _{1 \leq i \leq n}\left\|x_{i}\right\| \geq \sum_{j=1}^{n}\left\|x_{j}\right\|
\end{aligned}
$$

for all non-zero elements $x_{1}, \ldots, x_{n}$ of a normed linear space.

0 2013 Najati et al: licensee Springer. This is an Open Access article distributed under the terms of the Creative Commons Attribution License (http://creativecommons.org/licenses/by/2.0), which permits unrestricted use, distribution, and reproduction in any medium, provided the original work is properly cited. 
Pečarić and Rajić [5] sharpened inequalities (1.4) and (1.5) (when $n>2$ ) and generalized inequalities (1.2) and (1.3) by showing that

$$
\begin{aligned}
& \left\|\sum_{j=1}^{n} \frac{x_{j}}{\left\|x_{j}\right\|}\right\| \leq \min _{1 \leq i \leq n}\left\{\frac{1}{\left\|x_{i}\right\|}\left(\left\|\sum_{j=1}^{n} x_{j}\right\|+\sum_{j=1}^{n}\left|\left\|x_{j}\right\|-\left\|x_{i}\right\|\right|\right)\right\} \\
& \left\|\sum_{j=1}^{n} \frac{x_{j}}{\left\|x_{j}\right\|}\right\| \geq \max _{1 \leq i \leq n}\left\{\frac{1}{\left\|x_{i}\right\|}\left(\left\|\sum_{j=1}^{n} x_{j}\right\|-\sum_{j=1}^{n}\left|\left\|x_{j}\right\|-\left\|x_{i}\right\|\right|\right)\right\}
\end{aligned}
$$

for all non-zero elements $x_{1}, \ldots, x_{n}$ of a normed linear space.

Dragomir [6] replaced arbitrary scalars instead of $\frac{1}{\left\|x_{i}\right\|}$ for $i=1, \ldots, n$ in inequalities (1.6) and (1.7) and obtained a generalization of inequalities (1.6) and (1.7). This paper contains two sections. In the first section, we will work on a generalized case of Dunkl-Williams inequality in 2-normed spaces and also give necessary and sufficient conditions for having equality. In the second part, we want to introduce a refinement of the inequality $\| \frac{x}{\|x\|}-$ $\frac{y}{\|y\|} \| \leq \frac{2\|x-y\|}{\|x\|+\|y\|}$ in 2-inner product spaces, which was done by Mercer [3] in inner product spaces.

The concept of 2-normed spaces was introduced by Gähler [7] in 1963. After that, in 1973 and 1977, Diminnie, Gähler and White introduced the concept of 2-inner product spaces (see [8, 9]). We offer [10] to readers for more details.

Definition 1.1 Let $\mathbb{K}$ be the symbol of the field $\mathbb{R}$ or $\mathbb{C}$ and $X$ be a linear space on $\mathbb{K}$. Define the $\mathbb{K}$-valued function $(\cdot, \cdot \cdot \cdot)$ on $X \times X \times X$ with the following properties:

(1) $(x, x \mid y) \geq 0 ;(x, x \mid y)=0$ if and only if $x$ and $y$ are linearly dependent,

(2) $(x, x \mid y)=(y, y \mid x)$,

(3) $(x, y \mid z)=\overline{(y, x \mid z)}$

(4) $(\alpha x, y \mid z)=\alpha(x, y \mid z)$ for any $\alpha \in \mathbb{K}$,

(5) $\left(x+x^{\prime}, y \mid z\right)=(x, y \mid z)+\left(x^{\prime}, y \mid z\right)$,

for all $x, x^{\prime}, y, z \in X .(\cdot, \cdot \mid \cdot)$ is called a 2 -inner product and $(X,(\cdot, \cdot \mid \cdot))$ is called a 2-inner product space.

Lemma 1.2 [10] Let X be a 2-inner product space. Then

$$
|(x, y \mid z)| \leq \sqrt{(x, x \mid z)} \sqrt{(y, y \mid z)}
$$

for every $x, y, z \in X$.

Definition 1.3 [10] Let $X$ be a linear space of dimension greater than 1 on the field $\mathbb{K}$ and let $\|\cdot, \cdot\|: X \times X \rightarrow[0,+\infty)$ be a function satisfying the following conditions:

(1) $\|x, y\|=0$ if and only if $x$ and $y$ are linearly dependent,

(2) $\|x, y\|=\|y, x\|$,

(3) $\|\alpha x, y\|=|\alpha|\|x, y\|$ for all $\alpha \in \mathbb{K}$,

(4) $\|x+y, z\| \leq\|x, z\|+\|y, z\|$,

for all $x, y, z \in X .\|\cdot, \cdot\|$ is called a 2 -norm and $(X,\|\cdot, \cdot\|)$ is called a linear 2-normed space.

It follows from (4) that

$$
|\|x, z\|-\|y, z\|| \leq\|x-y, z\|
$$

for all $x, y, z \in X$. 
Let $X$ be a 2-inner product space of dimension greater than 1 on the field $\mathbb{R}$. If we define $\|x, y\|=\sqrt{(x, x \mid y)}$ for all $x, y \in X$, then $\|\cdot, \cdot\|$ is a 2-norm on $X$ and

$$
\begin{aligned}
& 4(x, y \mid z)=\|x+y, z\|^{2}-\|x-y, z\|^{2}, \\
& \|x+y, z\|^{2}+\|x-y, z\|^{2}=2\|x, z\|^{2}+2\|y, z\|^{2}
\end{aligned}
$$

(see Theorem 3.1.9 of [10]). In this case, (1.8) means

$$
|(x, y \mid z)| \leq\|x, z\|\|\mid\|, z \|
$$

A linear 2-normed space $(X,\|\cdot, \cdot\|)$ is said to be strictly convex if $\|x+y, z\|=\|x, z\|+\|y, z\|$, $\|x, z\|=\|y, z\|=1$ and $z \notin \operatorname{span}\{x, y\}$, then $x=y$.

\section{Main results}

In this section, we establish a generalization of Dunkl-Williams inequality and its reverse in 2-normed spaces.

Theorem 2.1 Let $X$ be a 2-normed space on the field $\mathbb{K}$. For $x_{1}, \ldots, x_{n}, z \in X$ and $a_{1}, \ldots, a_{n} \in$ $\mathbb{K}$, we have

$$
\begin{aligned}
\left\|\sum_{j=1}^{n} a_{j} x_{j}, z\right\| & \leq \min _{1 \leq i \leq n}\left\{\left|a_{i}\right|\left\|\sum_{j=1}^{n} x_{j}, z\right\|+\sum_{j=1}^{n}\left|a_{j}-a_{i}\right|\left\|x_{j}, z\right\|\right\}, \\
\left\|\sum_{j=1}^{n} a_{j} x_{j}, z\right\| & \geq \max _{1 \leq i \leq n}\left\{\left|a_{i}\right|\left\|\sum_{j=1}^{n} x_{j}, z\right\|-\sum_{j=1}^{n}\left|a_{j}-a_{i}\right|\left\|x_{j}, z\right\|\right\} .
\end{aligned}
$$

Proof For a fixed $1 \leq i \leq n$, we have

$$
\begin{aligned}
\left\|\sum_{j=1}^{n} a_{j} x_{j}, z\right\| & =\left\|\sum_{j=1}^{n} a_{i} x_{j}+\sum_{j=1}^{n}\left(a_{j}-a_{i}\right) x_{j}, z\right\| \\
& \leq\left\|\sum_{j=1}^{n} a_{i} x_{j}, z\right\|+\left\|\sum_{j=1}^{n}\left(a_{j}-a_{i}\right) x_{j}, z\right\| \\
& \leq\left\|\sum_{j=1}^{n} a_{i} x_{j}, z\right\|+\sum_{j=1}^{n}\left\|\left(a_{j}-a_{i}\right) x_{j}, z\right\| \\
& =\left|a_{i}\right|\left\|\sum_{j=1}^{n} x_{j}, z\right\|+\sum_{j=1}^{n}\left|a_{j}-a_{i}\right|\left\|x_{j}, z\right\| .
\end{aligned}
$$

By taking minimum over $i=1, \ldots, n$, we obtain $(2.1)$.

Now, we have

$$
\begin{aligned}
\left\|\sum_{j=1}^{n} a_{j} x_{j}, z\right\| & =\left\|\sum_{j=1}^{n} a_{i} x_{j}-\sum_{j=1}^{n}\left(a_{i}-a_{j}\right) x_{j}, z\right\| \\
& \geq\left\|\sum_{j=1}^{n} a_{i} x_{j}, z\right\|-\left\|\sum_{j=1}^{n}\left(a_{i}-a_{j}\right) x_{j}, z\right\|
\end{aligned}
$$




$$
\begin{aligned}
& \geq\left\|\sum_{j=1}^{n} a_{i} x_{j}, z\right\|-\sum_{j=1}^{n}\left\|\left(a_{i}-a_{j}\right) x_{j}, z\right\| \\
& =\left|a_{i}\right|\left\|\sum_{j=1}^{n} x_{j}, z\right\|-\sum_{j=1}^{n}\left|a_{i}-a_{j}\right|\left\|x_{j}, z\right\| .
\end{aligned}
$$

By taking maximum over $1 \leq i \leq n$, we obtain (2.2).

Theorem 2.2 [10] Let X be a real linear 2-normed space. The following statements are equivalent:

(1) $(X,\|\cdot, \cdot\|)$ is strictly convex.

(2) If $\|x+y, z\|=\|x, z\|+\|y, z\|$ and $z \notin \operatorname{span}\{x, y\}$, then $x=\lambda y$ for some $\lambda>0$.

Let $X$ be a real linear 2-normed space. If $z \notin \operatorname{span}\{x, y\}$ and $|(x, y \mid z)|=\|x, z\|\|y, z\|$, then $x=\lambda y$ for some real $\lambda$.

Lemma 2.3 Let $X$ be a linear strictly convex 2-normed space with respect to a 2-inner product on the field $\mathbb{R}$. For non-zero elements $x_{1}, \ldots, x_{n} \in X$ satisfying $\sum_{j=1}^{n} x_{j} \neq 0, z \notin$ $\operatorname{span}\left\{x_{1}, \ldots, x_{n}\right\}$ and non-zero elements $a_{1}, \ldots, a_{n}$ of $\mathbb{R}$ such that $a_{i} \neq a_{j}$ for some $i, j$, the following statements are equivalent:

(1) $\left\|\sum_{j=1}^{n} a_{j} x_{j}, z\right\|=\left|a_{i}\right|\left\|\sum_{j=1}^{n} x_{j}, z\right\|+\sum_{j=1}^{n}\left|a_{j}-a_{i}\right|\left\|x_{j}, z\right\|$;

(2) $\sum_{j=1}^{n}\left(x_{j}, x_{k} \mid z\right) a_{i}\left(a_{k}-a_{i}\right)=\left\|\sum_{j=1}^{n} x_{j}, z\right\|\left|a_{i}\right|\left|a_{k}-a_{i}\right|\left\|x_{k}, z\right\|$.

Proof Let (1) hold. For a fixed $1 \leq i \leq n$, we have

$$
\begin{aligned}
\left\|\sum_{j=1}^{n} a_{j} x_{j}, z\right\| & =\left\|\sum_{j=1}^{n} a_{i} x_{j}+\sum_{j=1}^{n}\left(a_{j}-a_{i}\right) x_{j}, z\right\| \\
& =\left|a_{i}\right|\left\|\sum_{j=1}^{n} x_{j}, z\right\|+\sum_{j=1}^{n}\left|a_{j}-a_{i}\right|\left\|x_{j}, z\right\| .
\end{aligned}
$$

From the assumption, there exists a non-empty maximal subset $\left\{j_{1}, \ldots, j_{m}\right\}$ of $\{1, \ldots, n\}$ for some $1 \leq m \leq n$ such that $a_{j_{k}} \neq a_{i}$ for all $1 \leq k \leq m$. Hence, (2.3) holds if and only if

$$
\left\|\sum_{j=1}^{n} a_{i} x_{j}+\sum_{k=1}^{m}\left(a_{j_{k}}-a_{i}\right) x_{j_{k}}, z\right\|=\left|a_{i}\right|\left\|\sum_{j=1}^{n} x_{j}, z\right\|+\sum_{k=1}^{m}\left|a_{j_{k}}-a_{i}\right|\left\|x_{j_{k}}, z\right\| .
$$

Using Theorem 2.2, we deduce that there exists $\beta_{j_{k}}>0$ such that $\sum_{j=1}^{n} a_{i} x_{j}=\beta_{j_{k}}\left(a_{j_{k}}-a_{i}\right) x_{j_{k}}$ which is equivalent to

$$
\left(\sum_{j=1}^{n} a_{i} x_{j},\left(a_{j_{k}}-a_{i}\right) x_{j_{k}} \mid z\right)=\left\|\sum_{j=1}^{n} a_{i} x_{j}, z\right\|\left\|\left(a_{j_{k}}-a_{i}\right) x_{j_{k}}, z\right\| .
$$

So, (1) and (2) are equivalent.

Lemma 2.4 Let $X$ be a linear strictly convex 2-normed space with respect to a 2-inner producton $\mathbb{R}$. For non-zero elements $x_{1}, \ldots, x_{n} \in X$ satisfying $\sum_{j=1}^{n} x_{j}=0, z \notin \operatorname{span}\left\{x_{1}, \ldots, x_{n}\right\}$ 
and non-zero elements $a_{1}, \ldots, a_{n}$ of $\mathbb{R}$ such that $a_{i} \neq a_{j}$ for some $i, j$, the following statements are equivalent:

(1) $\left\|\sum_{j=1}^{n} a_{j} x_{j}, z\right\|=\sum_{j=1}^{n}\left|a_{j}-a_{i}\right|\left\|x_{j}, z\right\|$.

(2) There exist $1 \leq i, l \leq n$ such that $a_{l} \neq a_{i}$ and

$\left(x_{k}, x_{l} \mid z\right)\left(a_{k}-a_{i}\right)\left(a_{l}-a_{i}\right)=\left\|x_{k}, z\right\|\left\|x_{l}, z\right\|\left|a_{k}-a_{i} \| a_{l}-a_{i}\right|$ for all $k$.

Proof Let $i(1 \leq i \leq n)$ be fixed. By $\sum_{j=1}^{n} x_{j}=0$, we get

$$
\sum_{j=1}^{n} a_{j} x_{j}=\sum_{j=1}^{n}\left(a_{j}-a_{i}\right) x_{j}
$$

Now, by the above equality and (1), we get

$$
\left\|\sum_{j=1}^{n}\left(a_{j}-a_{i}\right) x_{j}, z\right\|=\left\|\sum_{j=1}^{n} a_{j} x_{j}, z\right\|=\sum_{j=1}^{n}\left|a_{j}-a_{i}\right|\left\|x_{j}, z\right\| .
$$

Let $\left\{j_{1}, \ldots, j_{m}\right\}$ be as in the proof of Lemma 2.3. So,

$$
\left\|\sum_{k=1}^{m}\left(a_{j_{k}}-a_{i}\right) x_{j_{k}}, z\right\|=\sum_{k=1}^{m}\left|a_{j_{k}}-a_{i}\right|\left\|x_{j_{k}}, z\right\| .
$$

Now, let $1 \leq l \leq m$. Then we get

$$
\left\|\sum_{\substack{k=1 \\ k \neq l}}^{m}\left(a_{j_{k}}-a_{i}\right) x_{j_{k}}+\left(a_{j_{l}}-a_{i}\right) x_{j_{l}}, z\right\|=\sum_{\substack{k=1 \\ k \neq l}}^{m}\left|a_{j_{k}}-a_{i}\right|\left\|x_{j_{k}}, z\right\|+\left|a_{j_{l}}-a_{i}\right|\left\|x_{j_{l}}, z\right\| .
$$

Hence, for some $\beta_{j k}>0,\left(a_{j_{k}}-a_{i}\right) x_{j_{k}}=\beta_{j k}\left(a_{j_{l}}-a_{i}\right) x_{j_{l}}$. This is equivalent to

$$
\left(x_{j_{k}}, x_{j_{l}} \mid z\right)\left(a_{j_{k}}-a_{i}\right)\left(a_{j_{l}}-a_{i}\right)=\left\|x_{j_{k}}, z\right\|\left\|x_{j_{l}}, z\right\|\left|a_{j_{k}}-a_{i}\right|\left|a_{j_{l}}-a_{i}\right|
$$

as desired.

As an application of Lemmas 2.3 and 2.4, we offer the following theorem.

Theorem 2.5 Let $X$ be a linear strictly convex 2-normed space with respect to a 2-inner product on $\mathbb{R}$. Also, let $x_{1}, \ldots, x_{n} \in X$ be non-zero elements, $z \notin \operatorname{span}\left\{x_{1}, \ldots, x_{n}\right\}$ and $a_{1}, \ldots, a_{n} \in \mathbb{R}$ be non-zero elements such that $a_{i} \neq a_{j}$ for some $i, j$.

(1) If $\sum_{j=1}^{n} x_{j} \neq 0$, then

$$
\left\|\sum_{j=1}^{n} a_{j} x_{j}, z\right\|=\min _{1 \leq k \leq n}\left\{\left|a_{i}\right|\left\|\sum_{j=1}^{n} x_{j}, z\right\|+\sum_{j=1}^{n}\left|a_{j}-a_{i}\right|\left\|x_{j}, z\right\|\right\}
$$

if and only if there exists $1 \leq i \leq n$ such that

$$
\sum_{j=1}^{n}\left(x_{j}, x_{k} \mid z\right) a_{i}\left(a_{k}-a_{i}\right)=\left\|\sum_{j=1}^{n} x_{j}, z\right\|\left|a_{i}\right|\left|a_{k}-a_{i}\right|\left\|x_{k}, z\right\| .
$$


(2) If $\sum_{j=1}^{n} x_{j}=0$, then

$$
\left\|\sum_{j=1}^{n} a_{j} x_{j}, z\right\|=\min _{1 \leq k \leq n} \sum_{j=1}^{n}\left|a_{j}-a_{i}\right|\left\|x_{j}, z\right\|
$$

if and only if there exist $1 \leq i, l \leq n$ such that $a_{l} \neq a_{i}$ and

$$
\left(x_{k}, x_{l} \mid z\right)\left(a_{k}-a_{i}\right)\left(a_{l}-a_{i}\right)=\left\|x_{k}, z\right\|\left\|x_{l}, z\right\|\left|a_{k}-a_{i}\right|\left|a_{l}-a_{i}\right|
$$

for all $k$.

\section{Improvement of Dunkl-Williams inequality with two elements}

In [1], Dunkl and Williams proved that in inequality (1.1) the constant 4 is the best choice in normed linear spaces. Moreover, they proved that in an inner product space, the constant 4 can be replaced by 2 ; that is,

$$
\left\|\frac{x}{\|x\|}-\frac{y}{\|y\|}\right\| \leq \frac{2\|x-y\|}{\|x\|+\|y\|} .
$$

In addition, the equality in (3.1) holds if and only if $\|x\|=\|y\|$.

In 2 -inner product spaces, we have the following theorem.

Theorem 3.1 [11] For non-zero vectors $x, y, z$ in a 2-inner product space $X$ with $z \notin$ $\operatorname{span}\{x, y\}$

$$
\left\|\frac{x}{\|x, z\|}-\frac{y}{\|y, z\|}, z\right\| \leq \frac{2\|x-y, z\|}{\|x, z\|+\|y, z\|} .
$$

If $X$ is a linear 2-normed space in Theorem 3.1, then we have the following inequality:

$$
\left\|\frac{x}{\|x, z\|}-\frac{y}{\|y, z\|}, z\right\| \leq \frac{4\|x-y, z\|}{\|x, z\|+\|y, z\|} .
$$

A refinement of (3.1) has been obtained by Mercer [3]. Now, we use Mercer's inequality and give a refinement of (3.2).

Theorem 3.2 Let $x, y, z$ be non-zero vectors in a 2-inner product space $X$ with $z \notin$ $\operatorname{span}\{x, y\}$. Then we have

$$
\begin{aligned}
& \frac{(\|x, z\|-\|y, z\|)^{2}}{(\|x, z\|+\|y, z\|)^{2}}-\sqrt{\frac{4\|x-y, z\|^{2}}{(\|x, z\|+\|y, z\|)^{2}}+\frac{(\|x, z\|-\|y, z\|)^{4}}{(\|x, z\|+\|y, z\|)^{4}}-4 \frac{(\|x, z\|-\|y, z\|)^{2}}{(\|x, z\|+\|y, z\|)^{2}}} \\
& \leq\left\|\frac{x}{\|x, z\|}-\frac{y}{\|y, z\|}, z\right\| \\
& \leq \frac{(\|x, z\|-\|y, z\|)^{2}}{(\|x, z\|+\|y, z\|)^{2}} \\
& \quad+\sqrt{\frac{4\|x-y, z\|^{2}}{(\|x, z\|+\|y, z\|)^{2}}+\frac{(\|x, z\|-\|y, z\|)^{4}}{(\|x, z\|+\|y, z\|)^{4}}-4 \frac{(\|x, z\|-\|y, z\|)^{2}}{(\|x, z\|+\|y, z\|)^{2}}} .
\end{aligned}
$$


Proof Let $\alpha=\left\|\frac{x}{\|x, z\|}-\frac{y}{\|y, z\|}, z\right\|$. By using (2.2) for two elements $x,-y$ with constants $\frac{1}{\|x, z\|}$, $\frac{1}{\|y, z\|}$ respectively, we obtain

$$
\alpha \geq \frac{\|x-y, z\|-|\|x, z\|-\|y, z\||}{\min (\|x, z\|,\|y, z\|)} .
$$

Clearly, we have

$$
\begin{aligned}
& |\|y, z\|-\|x, z\||-\|x-y, z\|+2 \min (\|x, z\|,\|y, z\|) \\
& \quad=\|y, z\|+\|x, z\|-\|x-y, z\| .
\end{aligned}
$$

So,

$$
\|y, z\|+\|x, z\|-\|x-y, z\| \geq(2-\alpha) \min (\|x, z\|,\|y, z\|) .
$$

A simple computation shows that

$$
\frac{\operatorname{Re}(x, y \mid z)}{\|x, z\|\|y, z\|}=1-\frac{1}{2} \alpha^{2}, \quad \alpha^{2}=\frac{\|x-y, z\|^{2}-(\|y, z\|-\|x, z\|)^{2}}{\|x, z\|\|y, z\|} .
$$

Therefore,

$$
\begin{aligned}
\| x & -y, z \|^{2}-\left(\frac{\|y, z\|+\|x, z\|}{2}\right)^{2} \alpha^{2} \\
& =\frac{(\|y, z\|-\|x, z\|)^{2}}{4\|x, z\|\|y, z\|}\left((\|y, z\|+\|x, z\|)^{2}-\|x-y, z\|^{2}\right) \\
& =\frac{(\|y, z\|-\|x, z\|)^{2}}{4\|x, z\|\|y, z\|}(\|y, z\|+\|x, z\|-\|x-y, z\|)(\|y, z\|+\|x, z\|+\|x-y, z\|) \\
& \geq \frac{(\|y, z\|-\|x, z\|)^{2}}{4\|x, z\|\|y, z\|}(\|y, z\|+\|x, z\|-\|x-y, z\|)(\|y, z\|+\|x, z\|+\|\| x, z\|-\| y, z \|) \\
& =\frac{(\|y, z\|-\|x, z\|)^{2}}{2\|x, z\|\|y, z\|}(\|y, z\|+\|x, z\|-\|x-y, z\|) \max (\|x, z\|,\|y, z\|) \\
& \geq \frac{(\|y, z\|-\|x, z\|)^{2}}{2\|x, z\|\|y, z\|}(2-\alpha) \min (\|x, z\|,\|y, z\|) \max (\|x, z\|,\|y, z\|) \\
& =\frac{(\|y, z\|-\|x, z\|)^{2}}{2}(2-\alpha) .
\end{aligned}
$$

Hence,

$$
\|x-y, z\|^{2}-\left(\frac{\|y, z\|+\|x, z\|}{2}\right)^{2} \alpha^{2} \geq \frac{(\|y, z\|-\|x, z\|)^{2}}{2}(2-\alpha) .
$$

Therefore,

$$
\alpha^{2}-2 \alpha\left(\frac{\|y, z\|-\|x, z\|}{\|y, z\|+\|x, z\|}\right)^{2}+4 \frac{(\|y, z\|-\|x, z\|)^{2}-\|x-y, z\|^{2}}{(\|y, z\|+\|x, z\|)^{2}} \leq 0 .
$$


So, $\alpha$ is between two roots of the quadratic equation

$$
\lambda^{2}-2 \lambda\left(\frac{\|y, z\|-\|x, z\|}{\|y, z\|+\|x, z\|}\right)^{2}+4 \frac{(\|y, z\|-\|x, z\|)^{2}-\|x-y, z\|^{2}}{(\|y, z\|+\|x, z\|)^{2}}=0 .
$$

Hence, we get (3.3).

\section{Competing interests}

The authors declare that they have no competing interests.

\section{Authors' contributions}

All authors jointly worked on the results and they read and approved the final manuscript.

\section{Author details}

${ }^{1}$ Department of Mathematics, Faculty of Mathematical Sciences, University of Mohaghegh Ardabili, Ardabil, 56199-11367, Iran. ${ }^{2}$ Graduate School of Education, Kyung Hee University, Yongin, 446-701, Republic of Korea.

\section{Acknowledgements}

The authors wish to thank the anonymous referees for their valuable comments. This research was supported by the Basic Science Research Program through the National Research Foundation of Korea (NRF) funded by the Ministry of Education, Science and Technology (grant number 2012003499).

Received: 6 September 2012 Accepted: 17 January 2013 Published: 4 February 2013

\section{References}

1. Dunkl, CF, Williams, KS: A simple norm inequality. Am. Math. Mon. 71, $53-54$ (1964)

2. Maligranda, L: Simple norm inequalities. Am. Math. Mon. 113(3), 256-260 (2006)

3. Mercer, PG: The Dunkl-Williams inequality in an inner product space. Math. Inequal. Appl. 10(2), 447-450 (2007)

4. Kato, M, Saito, K-S, Tamura, T: Sharp triangle inequality and it's revers in Banach spaces. Math. Inequal. Appl. 10(2), 451-460 (2007)

5. Pečarić, JE, Rajić, R: The Dunkl-Williams inequality with $n$-elements in normed linear spaces. Math. Inequal. Appl. 10(2), 461-470 (2007)

6. Dragomir, SS: Generalization of the Pečarić-Rajić in normed linear spaces. Math. Inequal. Appl. 12(1), 53-65 (2009)

7. Gähler, S: 2-metrische Räume und ihre topologische struktur. Math. Nachr. 26, 115-148 (1963)

8. Diminnie, CR, Gähler, S, White, A: 2-inner product spaces. Demonstr. Math. 6, 525-536 (1973)

9. Diminnie, CR, Gähler, S, White, A: 2-inner product spaces II. Demonstr. Math. 10, 169-188 (1977)

10. Cho, YJ, Lin, CS, Kim, SS, Misiak, A: Theory of 2-Inner Product Spaces. Nova Science Publishers, New York (2001)

11. Cho, YJ, Dragomir, SS, Lin, CS, Kim, SS, Kim, YH: Some Pečarić's type inequality in 2-inner product spaces and applications. Taiwan. J. Math. 12(2), 257-372 (2008)

\section{Submit your manuscript to a SpringerOpen ${ }^{\circ}$ journal and benefit from:}

- Convenient online submission

- Rigorous peer review

- Immediate publication on acceptance

- Open access: articles freely available online

- High visibility within the field

- Retaining the copyright to your article 